\title{
Median eigenvalues of bipartite graphs
}

\author{
Bojan Mohar • Behruz Tayfeh-Rezaie
}

Received: 9 December 2013 / Accepted: 10 September 2014 / Published online: 24 September 2014 (C) Springer Science+Business Media New York 2014

\begin{abstract}
For a graph $G$ of order $n$ and with eigenvalues $\lambda_{1} \geqslant \cdots \geqslant \lambda_{n}$, the HL-index $R(G)$ is defined as $R(G)=\max \left\{\left|\lambda_{\lfloor(n+1) / 2\rfloor}\right|,\left|\lambda_{\lceil(n+1) / 2\rceil}\right|\right\}$. We show that for every connected bipartite graph $G$ with maximum degree $\Delta \geqslant 3, R(G) \leqslant \sqrt{\Delta-2}$ unless $G$ is the the incidence graph of a projective plane of order $\Delta-1$. We also present an approach through graph covering to construct infinite families of bipartite graphs with large HL-index.
\end{abstract}

Keywords Adjacency matrix - Graph eigenvalues $\cdot$ Median eigenvalues $\cdot$ Covers

Mathematics Subject Classification 05C50

\section{Introduction}

Unless explicitly stated, we assume that all graphs in this paper are simple, i.e. multiple edges and loops are not allowed. The adjacency matrix of $G$, denoted by $A(G)=$ $\left(a_{u v}\right)_{u, v \in V(G)}$, is a $(0,1)$-matrix whose rows and columns are indexed by the vertices of $G$ such that $a_{u v}=1$ if and only if $u$ is adjacent to $v$. We use $\operatorname{deg}_{G}(v)$ to denote the degree of a vertex $v$ in $G$. The set of all neighbours of $v$ is denoted by $N_{G}(v)$ and we write $N_{G}[v]=N_{G}(v) \cup\{v\}$. The smallest and largest degrees of $G$ are denoted by $\delta(G)$ and $\Delta(G)$, respectively.

B. Mohar

Department of Mathematics, Simon Fraser University, Burnaby, BC, Canada

e-mail: mohar@sfu.ca

B. Tayfeh-Rezaie $(\varangle)$

School of Mathematics, Institute for Research in Fundamental Sciences (IPM),

P.O. Box 19395-5746, Tehran, Iran

e-mail: tayfeh-r@ipm.ir 
Let $\lambda_{1} \geqslant \cdots \geqslant \lambda_{n}$ be the eigenvalues of $G$. Then $\lambda_{\lfloor(n+1) / 2\rfloor}$ and $\lambda_{\lceil(n+1) / 2\rceil}$ are called the median eigenvalues of $G$. These eigenvalues play an important role in mathematical chemistry since they are related to the HOMO-LUMO separation, see, e.g. [9] and [4,5]. Following [11], we define the $H L$-index $R(G)$ of the graph $G$ as

$$
R(G)=\max \left\{\left|\lambda_{\lfloor(n+1) / 2\rfloor}\right|,\left|\lambda_{\lceil(n+1) / 2\rceil}\right|\right\} .
$$

If $G$ is a bipartite graph, then $R(G)$ is equal to $\lambda_{n / 2}$ if $n$ is even and 0 , otherwise. In this paper, we show that for every connected bipartite graph $G$ with maximum degree $\Delta, R(G) \leqslant \sqrt{\Delta-2}$ unless $G$ is the the incidence graph of a projective plane of order $\Delta-1$, in which case it is equal to $\sqrt{\Delta-1}$. This extends the result of one of the authors [16] who proved the same for subcubic graphs.

On the other hand, we present an approach through graph covering to construct infinite families of connected graphs with large HL-index. More precisely, using coverings of the incidence graph of projective planes, we show that for any prime power $q$, there exist infinitely many connected bipartite $(q+1)$-regular graphs $G$ with $\sqrt{q-1}-1<R(G)<\sqrt{q}-1$. Graph coverings and analysis of their eigenvalues were instrumental in a recent breakthrough in spectral graph theory by Marcus, Spielman, and Srivastava who used graph coverings to construct infinite families of Ramanujan graphs of arbitrary degrees [14] and for solving the Kadison-Singer Conjecture [15]. In our paper, we find another application of a different character. As opposed to double covers used in [14], we use $k$-fold covering graphs with cyclic permutation representation and show that the behaviour of median eigenvalues can be controlled in certain instances. The main ingredient is a generalization of a result of Bilu and Linial [1] that eigenvalues of double covers over a graph $G$ are the union of the eigenvalues of $G$ and the eigenvalues of certain cover matrix $A^{-}$that is obtained from the adjacency matrix by replacing some of its entries by -1 . In our case, we use a family $A^{\lambda}$ of such matrices, where instead of -1 we use certain powers of a parameter $\lambda \in[-1,1]$. This result seems to be of independent interest.

\section{Bounds for bipartite graphs}

In this section, we obtain upper bounds on the HL-index of bipartite graphs in terms of maximum and minimum degrees of graphs. We consider regular graphs first.

Theorem 1 Let $G$ be a connected bipartite $k$-regular graph, where $k \geq 3$. If $R(G)>$ $\sqrt{k-2}$, then $R(G)=\sqrt{k-1}$ and $G$ is the incidence graph of a projective plane of order $k-1$.

Proof Let $|V(G)|=2 n$. The adjacency matrix of $G$ can be written as

$$
A(G)=\left[\begin{array}{cc}
0 & B \\
B^{T} & 0
\end{array}\right],
$$

where $B$ is a square matrix of order $n$. The matrix $E=B B^{T}-k I$ is a symmetric matrix of order $n$. Assuming that $R(G)>\sqrt{k-2}$, every eigenvalue $\lambda$ of $G$ satisfies 
$\lambda^{2}>k-2$ and hence all eigenvalues of $E$ are greater than -2 . Hence, $E+2 I$ is a positive definite matrix. All diagonal entries of this matrix are equal to 2 . Eigenvalue interlacing implies that any principal $2 \times 2$ submatrix of $E+2 I$ is positive definite, and therefore all off-diagonal entries are 0 or 1 . It follows that $E$ is the adjacency matrix of a graph $H$ with the least eigenvalue greater than -2 . We see that $H$ is regular since $E \mathbf{j}=\left(B B^{T}-k I\right) \mathbf{j}=\left(k^{2}-k\right) \mathbf{j}$. The connectedness of $G$ also yields that $H$ is connected. By Corollary 2.3.22 of [2], a connected regular graph with least eigenvalue greater than -2 is either a complete graph or an odd cycle. If $H$ is an odd cycle, then it is 2-regular and so from $k^{2}-k=2$, we have $k=2$, a contradiction. Hence $H$ is a complete graph. It is easy to see that this implies that $G$ is the incidence graph of a projective plane of order $k-1$.

For the next theorem, we need the following result [2, Theorem 2.3.20].

Theorem 2 ([2]) If $G$ is a connected graph with the least eigenvalue greater than -2, then one of the following holds:

(i) $G$ is the line graph of a multigraph $K$, where $K$ is obtained from a tree by adding one edge in parallel to a pendant edge;

(ii) $G$ is the line graph of a graph $K$, where $K$ is a tree or is obtained from a tree by adding one edge giving a nonbipartite unicyclic graph;

(iii) $G$ is one of the 573 exceptional graphs on at most 8 vertices.

We can now prove an analogue to Theorem 1 for non-regular graphs.

Theorem 3 Let $G$ be a connected bipartite nonregular graph with maximum degree $\Delta \geqslant 3$. Then $R(G) \leqslant \sqrt{\Delta-2}$.

Proof Let $d=\Delta-1$. Suppose, for a contradiction, that $R(G)>\sqrt{d-1}$. Let $\{U, W\}$ be the bipartition of $V(G)$. Then $U$ and $W$ have the same size, say $m$, since otherwise $R(G)$ would be zero. We proceed in the same way as in the proof of Theorem 1. The adjacency matrix of $G$ can be written in the form

$$
A(G)=\left[\begin{array}{cc}
0 & B \\
B^{T} & 0
\end{array}\right],
$$

where the rows of $B$ are indexed by the elements of $U$ and the columns by $W$. The matrix $E=B B^{T}-(d-1) I$ is a symmetric matrix of order $m$. Since $R(G)>\sqrt{d-1}$, we have $\lambda_{m}(E)>0$. Hence $E$ is a positive definite matrix whose diagonal entries are the integers $\operatorname{deg}_{G}(u)-(d-1) \leqslant 2, u \in U$. Since $E$ is positive definite, these are all equal to 1 or 2 and hence the degrees of vertices in $U$ are either $\Delta$ or $\Delta-1$. Moreover, this in turn implies that all off-diagonal entries of $E$ are either 0 or 1. Since the off-diagonal entries in $E$ count the number of walks of length 2 between vertices in $U$, the last conclusion in particular implies that $G$ has no 4-cycles. Let $D$ be the diagonal matrix whose diagonal is the same as the main diagonal of $E$. Let $H$ be the graph on $U$ with the adjacency matrix $A(H)=E-D$. Then the least eigenvalue of $H$ is greater than -2 and $A(H)+D$ is positive definite. The connectedness of $G$ yields that $H$ is connected. 
Suppose that $v_{1}, v_{2} \in U$ are distinct vertices of degree $d$ in $G$. Let $P$ be a shortest path in $H$ connecting $v_{1}$ to $v_{2}$. The vertices $v_{1}, v_{2}$ and the path $P$ can be chosen so that all internal vertices on $P$ are of degree $d+1$ in $G$. Then $A(P)+\operatorname{diag}(1,2, \ldots, 2,1)$, which is a principal submatrix of $A(H)+D$, has eigenvalue 0 with the eigenvector $(1,-1,1,-1, \ldots)^{T}$, a contradiction. This shows that $U$ contains at most one vertex which has degree $d$ in $G$. Note that the same argument can be applied to $W$. As $G$ is not regular, we conclude that it has precisely two vertices of degree $d$, one in $U$ and one in $W$. Therefore, we may assume that $D=\operatorname{diag}(2, \ldots, 2,1)$.

Let us now consider degrees of vertices in $H$. Since $G$ has no 4-cycles, we have for every $u \in U$ :

$$
\operatorname{deg}_{H}(u)=\sum_{u v \in E(G)}\left(\operatorname{deg}_{G}(v)-1\right)
$$

Since $U$ and $W$ each has precisely one vertex whose degree in $G$ is $d$, (1) implies the following: If $\operatorname{deg}_{G}(u)=d$, then $\operatorname{deg}_{H}(u) \in\left\{d^{2}, d^{2}-1\right\}$; if $\operatorname{deg}_{G}(u)=d+1$, then $\operatorname{deg}_{H}(u) \in\left\{d^{2}+d, d^{2}+d-1\right\}$ with the smaller value only when $u$ is adjacent to the vertex of degree $d$ in $W$. Thus, $H$ has a unique vertex of degree $d^{2}$ or $d^{2}-1$, at most $d$ vertices of degree $d^{2}+d-1$, and all other vertices are of degree $d^{2}+d$.

Let $v \in U$ be the vertex with $\operatorname{deg}_{G}(v)=d$. We claim that the neighbourhood of $v$ in $H$ is a complete graph. This is since

$$
\left[\begin{array}{lll}
2 & 0 & 1 \\
0 & 2 & 1 \\
1 & 1 & 1
\end{array}\right]
$$

is not positive definite and so it cannot be a principal submatrix of $A(H)+D$. We also claim that $N_{H}\left[v_{1}\right] \cap N_{H}\left[v_{2}\right] \subseteq N_{H}[v]$ for arbitrary distinct vertices $v_{1}, v_{2} \in N_{H}(v)$. If this were not the case, the matrix

$$
\left[\begin{array}{llll}
2 & 1 & 1 & 0 \\
1 & 2 & 1 & 1 \\
1 & 1 & 2 & 1 \\
0 & 1 & 1 & 1
\end{array}\right]
$$

which is not positive definite, would be a principal submatrix of $A(H)+D$, a contradiction. Therefore, $H$ has at least $1+\left(d^{2}-1\right)+\left(d^{2}-1\right)\left(\left(d^{2}+d-1\right)-\left(d^{2}-1\right)\right)=$ $d^{3}+d^{2}-d$ or $1+d^{2}+d^{2}\left(\left(d^{2}+d-1\right)-d^{2}\right)=d^{3}+1$ vertices. In any case, we have $|V(H)| \geqslant 9$ and so $H$ is the case (i) or (ii) of Theorem 2 .

Let $H$ be the line graph of a multigraph $K$ as in Theorem 2, where $|V(K)|=m$ or $m+1$ and $|E(K)|=m$. Note that $K$ is not a cycle, since then $H$ would be regular in that case. So, from $\sum_{v \in V(K)} \operatorname{deg}_{K}(v)=2 m$ and $|V(K)| \geqslant m$, we have a vertex $u$ such that $\operatorname{deg}_{K}(u)=1$. Let $u^{\prime}$ be the unique neighbour of $u$ in $K$. The degree of the vertex in $H$ corresponding to the edge $u u^{\prime}$ is at least $d^{2}-1$. Thus, $\operatorname{deg}_{K}(u)+\operatorname{deg}_{K}\left(u^{\prime}\right)-2 \geqslant d^{2}-1$ and so $\operatorname{deg}_{K}\left(u^{\prime}\right) \geqslant d^{2}$. Let $r$ be the number of vertices of degree one in $K$. The sum of degrees in $K$ is at least $r+d^{2}+2(|V(K)|-r-1) \leqslant 2 m$ which gives $r \geqslant d^{2}-2 \geqslant 2$. Since $H$ has only one vertex of degree $d^{2}-1$ or $d^{2}$, we may take $u$ to be a vertex of 
degree 1 in $K$ such that $\operatorname{deg}_{K}(u)+\operatorname{deg}_{K}\left(u^{\prime}\right)-2 \geqslant d^{2}+d-1$ and now a similar argument as above gives $r \geqslant d^{2}+d-2>d+1$. Now again since $H$ has at most $d+1$ vertices of degree $d^{2}-1, d^{2}$ or $d^{2}+d-1$, we may take $u$ such that $\operatorname{deg}_{K}(u)+\operatorname{deg}_{K}\left(u^{\prime}\right)-2=d^{2}+d$. It follows that $H$ has the complete graph of order $d^{2}+d+1$ as a subgraph which in turn implies that $H$ is in fact the complete graph of order $d^{2}+d+1$ since $H$ is connected with maximum degree $d^{2}+d$. And this contradicts the fact that $H$ is nonregular.

Theorems 1 and 3 can be combined into our main result.

Theorem 4 Let $G$ be a connected bipartite graph with maximum degree $\Delta \geqslant 3$. Then $R(G) \leqslant \sqrt{\Delta-2}$ unless $G$ is the the incidence graph of a projective plane of order $\Delta-1$, in which case $R(G)=\sqrt{\Delta-1}$.

There are connected graphs that are not incidence graphs of projective planes and attain the bound $\sqrt{\Delta-2}$ of Theorem 4 . The incidence graph of a biplane $((v, k, 2)$ symmetric design) has degree $k$ and HL-index $\sqrt{k-2}$. Only 17 biplanes are known and the question of existence of infinitely many biplanes is an old open problem in design theory [10]. An infinite family of cubic graphs with HL-index equal to 1 is constructed in [8].

Finally, we give an upper bound for the HL-index of bipartite graphs in term of the minimum degree.

Theorem 5 Let $G$ be a bipartite graph with minimum degree $\delta$. Then $R(G) \leqslant \sqrt{\delta}$. Moreover, if $\delta \geq 2$ or $\delta=1$ and $G$ has a component with minimum degree 1 that is not isomorphic to $K_{2}$, then $R(G)<\sqrt{\delta}$.

Proof We may assume that $G$ is connected and of even order $n=2 m$. Let $v$ be a vertex of degree $\delta$ and $H=G-v$. Since $G$ is connected, $\lambda_{1}(G)>\lambda_{1}(H)$. By interlacing, $\lambda_{i}(G) \geqslant \lambda_{i}(H)$ for $1<i \leq m$. We also have $\lambda_{m}(H)=0$ since $H$ is bipartite and has an odd number of vertices.

The sum of the squares of the eigenvalues of $G$ is the trace of $A^{2}$, which is equal to $2|E(G)|$. By considering only half of the eigenvalues and using the fact that eigenvalues of a bipartite graph are symmetric about zero, we have:

$$
\begin{aligned}
\lambda_{m}^{2}(G) & =|E(G)|-\sum_{i=1}^{m-1} \lambda_{i}^{2}(G) \\
& =\delta+\sum_{i=1}^{m-1} \lambda_{i}^{2}(H)-\sum_{i=1}^{m-1} \lambda_{i}^{2}(G) \\
& \leqslant \delta .
\end{aligned}
$$

If $m \geqslant 2$, then for $i=1$, we have $\lambda_{1}^{2}(H)-\lambda_{1}^{2}(G)<0$, so the last inequality is strict. This proves the assertion of the theorem. 


\section{Covering graphs and their eigenvalues}

A covering map $f$ from a graph $\hat{G}$ to another graph $G$ is a surjection and a local isomorphism: the neighbourhood of a vertex $v$ in $\hat{G}$ is mapped bijectively onto the neighbourhood of $f(v)$ in $G$ (see [6, page 115]). $\hat{G}$ is said to be a covering graph (also called a lift) of $G$. All eigenvalues of $G$ are included in the spectrum of $\hat{G}$. The essence of this section is to show how to control the newly arising eigenvalues in the covering graph.

We will denote the eigenvalues of a symmetric $n \times n$ matrix $M$ by $\lambda_{1}(M) \geqslant \cdots \geqslant$ $\lambda_{n}(M)$. Also, if $\mathbf{x}$ is an eigenvector of $M$, then we denote the corresponding eigenvalue by $\lambda_{\mathbf{x}}(M)$. For a positive integer $t$, let $I_{t}$ and $\mathbf{0}_{t}$ denote the $t \times t$ identity matrix and the $t \times t$ all-zero matrix, respectively. A permutation matrix $C$ of size $t$ and order $m$ is a $t \times t(0,1)$-matrix that has exactly one entry 1 in each row and each column and $m$ is the smallest positive integer such that $C^{m}=I_{t}$.

Let us replace each edge of a multigraph $G$ by two oppositely oriented directed edges joining the same pair of vertices, and let $\vec{E}(G)$ denote the resulting set of directed edges. We denote by $(e, u, v) \in \vec{E}(G)$ the directed edge in $\vec{E}(G)$ corresponding to an edge $e=u v$ that is oriented from $u$ to $v$. Let $S_{t}$ denote the symmetric group of all permutations of size $t$. We shall consider a representation of $S_{t}$ as the set of all permutation matrices of size $t$. A function $\phi: \vec{E}(G) \rightarrow S_{t}$ is a permutation voltage assignment for $G$ if $\phi(e, u, v)=\phi(e, v, u)^{-1}$ for every $e \in E(G)$. A $t$-lift of $G$ associated to $\phi$ and denoted by $G(\phi)$ is a multigraph with the adjacency matrix obtained from the adjacency matrix of $G$ by replacing any $(u, v)$-entry of $A(G)$ by the $t \times t$ matrix $\sum_{(e, u, v) \in \vec{E}(G)} \phi(e, u, v)$. Note that if $G$ is bipartite, then so is $G(\phi)$. Also if $G$ is $k$-regular, then so is $G(\phi)$. We say that $G(\phi)$ is an Abelian lift if all matrices in the image of $\phi$ commute with each other. For more on voltage assignments, we refer the reader to [7] and the references therein.

Bilu and Linial [1] found an expression for the spectrum of 2-lifts. They proved that the spectrum of a 2-lift $G(\phi)$ consists of the spectrum of $G$ together with the spectrum of the matrix $A^{-}$which is obtained from the adjacency matrix of $G$ by replacing each $(u, v)$-entry by -1 whenever the voltage $\phi(e, u, v)$ is not the identity. Note that 2-lifts are always Abelian since the permutation matrices of size 2 commute with each other. Below we extend the result of [1] to arbitrary Abelian $t$-lifts. Since permutation matrices are diagonalizable and any commuting family of diagonalizable $t \times t$ matrices has a common basis of eigenvectors, we observe that any commuting set of permutation matrices of the same size has a common basis of eigenvectors.

In the proofs we will use the following result, see [12, Theorem 1].

Theorem 6 ([12]) Let $t$ and $n$ be positive integers and for $i, j \in\{1, \ldots, n\}$, let $B_{i j}$ be $t \times t$ matrices over a commutative ring that commute pairwise. Then

$$
\operatorname{det}\left[\begin{array}{ccc}
B_{11} & \cdots & B_{1 n} \\
\vdots & \ddots & \vdots \\
B_{n 1} & \cdots & B_{n n}
\end{array}\right]=\operatorname{det}\left(\sum_{\sigma \in S_{n}} \operatorname{sign}(\sigma) B_{1 \sigma(1)} \cdots B_{n \sigma(n)}\right),
$$

where $S_{n}$ is the set of all permutations of $\{1, \ldots, n\}$. 
Theorem 7 Let $G$ be a multigraph and $\phi$ be a permutation voltage assignment for an Abelian t-lift $G(\phi)$ of $G$. Let $\mathcal{B}$ be a common basis of eigenvectors of the permutation matrices in the image of $\phi$. For every $\mathbf{x} \in \mathcal{B}$, let $A_{\mathbf{x}}$ be the matrix obtained from the adjacency matrix of $G$ by replacing any $(u, v)$-entry of $A(G)$ by $\sum_{(e, u, v) \in \vec{E}(G)} \lambda_{\mathbf{x}}(\phi(e, u, v))$. Then the spectrum of $G(\phi)$ is the multiset union of the spectra of the matrices $A_{\mathbf{x}}(\mathbf{x} \in \mathcal{B})$.

Proof The adjacency matrix of a $t$-lift can be written in the block form, with the blocks being indexed by $V(G)$, where the $(u, v)$-block $D_{u v}$ is equal to the permutation matrix $\phi(e, u, v)$ if $u, v$ are joined by a single edge $e$, or to $\sum_{(e, u, v) \in \vec{E}(G)} \phi(e, u, v)$ if there are multiple edges, or $\mathbf{0}_{t}$ if $u, v$ are not adjacent in $G$. Thus, assuming $V(G)=\{1, \ldots, n\}$, we can write

$$
\lambda I-A(G(\phi))=\left[\begin{array}{ccc}
B_{11} & \cdots & B_{1 n} \\
\vdots & \ddots & \vdots \\
B_{n 1} & \cdots & B_{n n}
\end{array}\right]
$$

where the diagonal blocks are $\lambda I_{t}$, while the off-diagonal blocks are $B_{u v}=-D_{u v}$. All block matrices $B_{u v}$ commute with each other and all their products and sums also commute and have $\mathcal{B}$ as a common basis of eigenvectors. By Theorem 6, we have

$$
\begin{aligned}
\operatorname{det}(\lambda I-A(G(\phi))) & =\operatorname{det}\left(\sum_{\sigma \in S_{n}} \operatorname{sign}(\sigma) B_{1 \sigma(1)} \cdots B_{n \sigma(n)}\right) \\
& =\prod_{\mathbf{x} \in \mathcal{B}} \lambda_{\mathbf{x}}\left(\sum_{\sigma \in S_{n}} \operatorname{sign}(\sigma) B_{1 \sigma(1)} \cdots B_{n \sigma(n)}\right) \\
& =\prod_{\mathbf{x} \in \mathcal{B}}\left(\sum_{\sigma \in S_{n}} \operatorname{sign}(\sigma) \lambda_{\mathbf{x}}\left(B_{1 \sigma(1)}\right) \cdots \lambda_{\mathbf{x}}\left(B_{n \sigma(n)}\right)\right) \\
& =\prod_{\mathbf{x} \in \mathcal{B}} \operatorname{det}\left(\lambda I-A_{\mathbf{x}}\right) .
\end{aligned}
$$

This equality gives the conclusion of the theorem.

Let us keep the notation of Theorem 7 and its proof. There are two things to be observed. Since every permutation matrix $D_{u v}$ satisfies $D_{u v}^{-1}=D_{u v}^{T}$, we have that the matrices $A_{\mathbf{x}}(\mathbf{x} \in \mathcal{B})$ are Hermitian. The $(u, v)$-entry of $A_{\mathbf{x}}$ is the eigenvalue of $D_{u v}$ corresponding to the eigenvector $\mathbf{x}$. Thus the characteristic polynomial $\varphi\left(A_{\mathbf{x}}, \lambda\right)=$ $\operatorname{det}\left(\lambda I-A_{\mathbf{x}}\right)$ is a polynomial in $\lambda$, whose coefficients are polynomials in the $\mathbf{x}$ eigenvalues of the permutation matrices in the image of $\phi$.

\section{Median eigenvalues of covering graphs}

In this section we present an approach through graph covering to construct infinite families of graphs with large HL-index. The main tool is Theorem 7, which will be invoked in a very special situation. 
Let us select a set $F \subseteq \vec{E}(G)$ of oriented edges such that whenever $(e, u, v) \in F$, the opposite edge $(e, v, u)$ is not in $F$. For every positive integer $t$, let $C_{t}$ be a cyclic permutation of size and order $t$. Now, let us consider an infinite family of Abelian lifts $\phi_{1}, \phi_{2}, \phi_{3}, \ldots$ such that $\phi_{t}$ is an Abelian $t$-lift over the graph $G$, whose voltages are given by the following rule:

$$
\phi_{t}(e, u, v)=\left\{\begin{array}{l}
C_{t} \text { if }(e, u, v) \text { in } F \\
C_{t}^{-1} \text { if }(e, v, u) \text { in } F \\
I_{t} \text { otherwise. }
\end{array}\right.
$$

In this way, we obtain an infinite family of graphs $G\left(\phi_{t}\right)$. By Theorem 7, we can express the characteristic polynomial of $A\left(G\left(\phi_{t}\right)\right)$ as a product of the characteristic polynomials of matrices $A_{\mathbf{x}}$. For each $\mathbf{x} \in \mathcal{B}$, the characteristic polynomial of $A_{\mathbf{x}}$ depends only on $\lambda$ and on the eigenvalue $\alpha=\lambda_{\mathbf{x}}\left(C_{t}\right)$ and on $\lambda_{\mathbf{x}}\left(C_{t}^{-1}\right)=\alpha^{-1}=\bar{\alpha}$. The dependence on $\alpha$ can be expressed in terms of the real parameter $v=\alpha+\bar{\alpha}$. For cyclic permutations, every such $v$ is an eigenvalue of the $t$-cycle, which is of the form $v=2 \cos (2 \pi j / t)$ for some $j \in\{0,1, \ldots, t-1\}$. Thus, there is a polynomial $\Phi(\lambda, v)$ such that

$$
\operatorname{det}\left(\lambda I-A\left(G\left(\phi_{t}\right)\right)=\prod_{0 \leqslant j<t} \Phi(\lambda, 2 \cos (2 \pi j / t)) .\right.
$$

Note that $\Phi(\lambda, v)$ is independent of $t$ and only depends on the underlying graph $G$ and the values for $v$ lie in the interval $[-2,2]$ for every $t$. All eigenvalues of $G\left(\phi_{t}\right)$ correspond to the zero-set of the polynomial $\Phi(\lambda, v)$ with $v \in[s-2,2]$. When $t$ gets large, the appropriate values of $v$ become dense in the interval $[-2,2]$. This shows that if $G$ is bipartite, then $R\left(G\left(\phi_{t}\right)\right)$ converges to some value when $t$ goes to infinity. This is better seen in a special case which is given in the following theorem.

Theorem 8 Let $G$ be a bipartite graph and let $E_{0}$ be a set of edges all incident with some fixed vertex $v_{0}$. Let $F \subset \vec{E}(G)$ be the set of directed edges $\left\{\left(e, u, v_{0}\right) \mid u v_{0} \in\right.$ $\left.E_{0}\right\}$. For each positive integer $t$, fix a cyclic permutation matrix $C_{t}$ of size and order $t$ and define a permutation voltage assignment $\phi_{t}$ by (2). Then

$$
R\left(G\left(\phi_{2 t}\right)\right)=R\left(G\left(\phi_{2}\right)\right)
$$

for every $t \geq 1$, whereas the values $R\left(G\left(\phi_{2 t+1}\right)\right)$ are non-increasing as a function of $t$ and

$$
\lim _{t \rightarrow \infty} R\left(G\left(\phi_{2 t+1}\right)\right)=R\left(G\left(\phi_{2}\right)\right) .
$$

Proof By Theorem 7, there is a polynomial $\Phi(\lambda, v)$ such that (3) holds and every eigenvalue of any $G\left(\phi_{t}\right)$ lies among the values $\lambda$ for which there is a $\nu \in[-2,2]$ such that $\Phi(\lambda, v)=0$. It is easy to see that our choice of $F$ implies that $\Phi(\lambda, v)$ is linear in $v$, so it can be expressed in the form

$$
\Phi(\lambda, v)=p(\lambda)-v q(\lambda) .
$$


If $R\left(G\left(\phi_{2}\right)\right)$ is zero, then $R\left(G\left(\phi_{2 t}\right)\right)=0$ for every $t \geq 1$, since by Theorem 7 , the spectrum of $G\left(\phi_{2}\right)$ is contained in the spectrum of $G\left(\phi_{2 t}\right)$. Hence, we may assume that $R\left(G\left(\phi_{2}\right)\right) \neq 0$. Note that $R(G) \geq R\left(G\left(\phi_{2}\right)\right)$, so we also have $R(G) \neq 0$.

Let us first assume that $q(0) \neq 0$. Let $\Phi\left(0, v_{0}\right)=0$. Then $v_{0}=p(0) / q(0)$. We have $\Phi(0,2)=p(0)-2 q(0)$ and $\Phi(0,-2)=p(0)+2 q(0)$ which results in

$$
v_{0}=p(0) / q(0)=\frac{2(\Phi(0,-2)+\Phi(0,2))}{\Phi(0,-2)-\Phi(0,2)} .
$$

On the other hand, Eq.(3) gives that $\Phi(0,2)=\operatorname{det}(-A(G))$ and $\Phi(0,-2)=$ $\operatorname{det}\left(-A\left(G\left(\phi_{2}\right)\right)\right) / \operatorname{det}(-A(G))$. Since the eigenvalues of bipartite graphs $G$ and $G\left(\phi_{2}\right)$ are symmetric about zero, this implies that the above determinants, and thus also $\Phi(0,2)$ and $\Phi(0,-2)$, have the same sign. It follows that $\left|v_{0}\right|>2$. Since $\Phi(\lambda, v)$ is linear in $v$, for each $\lambda$ there exists at most one value $v$ such that $\Phi(\lambda, v)=0$ (and there is exactly one if $q(\lambda) \neq 0)$. Therefore, the continuity of $\Phi(\lambda, v)$ and its linearity in $v$ show that the eigenvalue $R\left(G\left(\phi_{t}\right)\right)$ is either a root of $\Phi(\lambda, 2)$ or a root of $\Phi(\lambda,-2)$ (if $t$ is even) or a root of $\Phi(\lambda, 2 \cos (\pi(t-1) / t)$ ) (if $t$ is odd). This is independent of $t$ when $t$ is even and is already among the eigenvalues of $G\left(\phi_{2}\right)$. For odd values of $t$, this shows the behaviour as claimed in the theorem.

Suppose next that $q(0)=0$. Then $p(0) \neq 0$, since otherwise we have $\Phi(0,2)=0$ and so $R(G)=0$, a contradiction. This shows that if $\Phi\left(\lambda_{0}, \nu_{0}\right)=0$ and $\lambda_{0}$ goes to zero, then $v_{0}$ goes to infinity. Again the continuity of $\Phi(\lambda, v)$ and its linearity in $v$ show that $R\left(G\left(\phi_{t}\right)\right)$ is either a root of $\Phi(\lambda, 2)$ or a root of $\Phi(\lambda,-2)$ (if $t$ is even) or a root of $\Phi(\lambda, 2 \cos (\pi(t-1) / t)$ ) (if $t$ is odd). We now complete the proof in the same as above.

Theorem 9 For any integer $k$ for which $k-1$ is a prime power, there exist infinitely many connected bipartite $k$-regular graphs $G$ with $\sqrt{k-2}-1<R(G)<\sqrt{k-1}-1$.

Proof Let $G$ be the incidence graph of a projective plane of order $q=k-1$. Note that $G$ is bipartite and $k$-regular. It is well-known (see, e.g., [6]) that $G$ has eigenvalues $\pm k$ and $\pm \sqrt{q}$. Thus, $R(G)=\sqrt{q}$. Let $e_{0}$ be any edge of $G$ and $E_{0}=\left\{e_{0}\right\}$. For each positive integer $t$, define the permutation assignment $\phi_{t}$ as in Theorem 8 .

The adjacency matrix of $G\left(\phi_{2}\right)$ can be written as $A\left(G\left(\phi_{2}\right)\right)=\left(A(G) \otimes I_{2}\right)+B$, where $B$ is a matrix with only \pm 2 as nonzero eigenvalues. Let $r$ be the number of vertices of $G\left(\phi_{2}\right)$. By the Courant-Weyl inequalities $\lambda_{i+j-r}(M+N) \geqslant \lambda_{i}(M)+\lambda_{j}(N)$ for generic square matrices $M$ and $N$ of the same order, we have $\lambda_{r / 2-1}\left(A\left(G\left(\phi_{2}\right)\right)\right) \geqslant$ $\lambda_{r / 2}\left(A(G) \otimes I_{2}\right)+\lambda_{r-1}(B)$ which gives $\lambda_{r / 2-1}\left(G\left(\phi_{2}\right)\right) \geqslant \sqrt{q}$. In fact, since $G\left(\phi_{2}\right)$ has $\sqrt{q}$ as an eigenvalue with big multiplicity, one observes that $\lambda_{r / 2-1}\left(G\left(\phi_{2}\right)\right)=\sqrt{q}$ and so $R(G)=\lambda_{r / 2}\left(G\left(\phi_{2}\right)\right) \leqslant \sqrt{q}$.

Let $e_{0}=\left\{v_{0}, v_{1}\right\}$. Let us consider the partition $\left\{v_{0}, v_{1}\right\} \cup W \cup W^{\prime}$ of $V(G)$, where $W$ is the set of all vertices adjacent to $v_{0}$ or $v_{1}$ and $W^{\prime}$ is the set of vertices nonadjacent to $v_{0}, v_{1}$. Define the vector $\mathbf{x}$ on $V(G)$ as

$$
\mathbf{x}(w)=\left\{\begin{array}{l}
1 \text { if } w=v_{0} \text { or } w=v_{1}, \\
a \text { if } w \text { in } W \\
b \text { if } w \text { in } W^{\prime}
\end{array}\right.
$$


We now consider the vector $\mathbf{y}=(\mathbf{x},-\mathbf{x})$ on $V\left(G\left(\phi_{2}\right)\right)$. Since the girth of $G$ is six, it is easy to see that $\mathbf{y}$ is an eigenvector of $G\left(\phi_{2}\right)$ with the corresponding eigenvalue $\lambda$ if and only if $\lambda=q a-1, a \lambda=q b+1$, and $b \lambda=q b+a$.

Solving these equations in term of $\lambda$ gives $\lambda^{3}+(1-q) \lambda^{2}-3 q \lambda+q^{2}-q=0$. The value of the expression on the left side of this equation is 2 and $\sqrt{q}-q$ for $\lambda=\sqrt{q-1}-1$ and $\lambda=\sqrt{q}-1$, respectively. Therefore, there is a root between $\sqrt{q-1}-1$ and $\sqrt{q}-1$. This implies that $\sqrt{q-1}-1<R\left(G\left(\phi_{2}\right)\right)<\sqrt{q}-1$. Finally, since $R(G)=\sqrt{q}$, Theorem 8 implies that $\sqrt{q-1}-1<R\left(G\left(\phi_{2 t}\right)\right)=$ $R\left(G\left(\phi_{2}\right)\right)<\sqrt{q}-1$ for all $t$.

The proof of Theorem 9 can be used to obtain a slightly better bound on $R(G)$. Let $t=\sqrt{k-1}$. The value of $\lambda^{3}+(1-q) \lambda^{2}-3 q \lambda+q^{2}-q$ is

$$
(h t)^{-3}\left(\left(2 h^{2}-h^{3}\right) t^{5}+\left(h^{3}-2 h^{2}\right) t^{4}+\left(4 h^{2}-h\right) t^{3}+\left(3 h-h^{2}\right) t^{2}-2 h t-1\right)
$$

for $\lambda=t-1-(h t)^{-1}$. Note that (4) is positive for $h=2$ and any $t$, whereas it is negative for any $h>2$ if $t$ is large enough. Therefore we find that $\sqrt{k-1}-1-\frac{1}{2 \sqrt{k-1}}<$ $R(G)<\sqrt{k-1}-1-\frac{1}{(2+\epsilon) \sqrt{k-1}}$ for every $\epsilon>0$ and any large $k$.

The authors were informed that Theorem 7 was obtained previously by Kwak and Lee in [13]. A generalization is also given in [3].

Acknowledgments The authors are grateful to anonymous referees for their valuable comments and remarks. Bojan Mohar:Supported in part by an NSERC Discovery Grant (Canada), by the Canada Research Chair program, and by the Research Grant P1-0297 of ARRS (Slovenia). On leave from: IMFM \& FMF, Department of Mathematics, University of Ljubljana, Ljubljana,Slovenia

\section{References}

1. Bilu, Y., Linial, N.: Lifts, discrepancy and nearly optimal spectral gap. Combinatorica 26, 495-519 (2006)

2. Cvetković, D., Rowlinson, P., Simić, S.: Spectral generalizations of line graphs, On graphs with least eigenvalue 2. London Mathematical Society Lecture Notes Series 314. Cambridge University Press, Cambridge (2004)

3. Feng, R., Kwak, J.H., Lee, J.: Characteristic polynomials of graph coverings. B. Aust. Math. Soc. 69, 133-136 (2004)

4. Fowler, P.W., Pisanski, T.: HOMO-LUMO maps for fullerenes. Acta Chim. Slov. 57, 513-517 (2010)

5. Fowler, P.W., Pisanski, T.: HOMO-LUMO maps for chemical graphs. MATCH Commun. Math. Comput. Chem. 64, 373-390 (2010)

6. Godsil, C., Royle, G.: Algebraic Graph Theory. Springer, Berlin (2001)

7. Gross, J.L.: Voltage Graphs. In: Jonathan, L. (ed.) Handbook of Graph Theory, Discrete Mathematics and its Applications, pp. 783-805. CRC Press, Boca Raton (2004)

8. Guo, K., Mohar, B.: Large regular bipartite graphs with median eigenvalue 1, submitted. arXiv: 1309.7025

9. Gutman, I., Polanski, O.E.: Mathematical Concepts in Organic Chemistry. Springer-Verlag, Berlin (1986)

10. Hall, M.: Combinatorial Theory, 2nd edn. Wiley, Hoboken (1986)

11. Jaklič, G., Fowler, P.W., Pisanski, T.: HL-index of a graph. Ars Math. Contemp. 5, 99-105 (2012)

12. Kovacs, I., Silver, D.S., Williams, S.G.: Determinants of commuting-block matrices. Amer. Math. Monthly 106, 950-952 (1999)

13. Kwak, J.H., Lee, J.: Characteristic polynomials of some graph bundles II. Linear Multilinear A. 32, 61-73 (1992) 
14. Marcus, A., Spielman, D.A., Srivastava, N.: Interlacing families I: Bipartite Ramanujan graphs of all degrees, arXiv:1304.4132

15. Marcus, A., Spielman, D.A., Srivastava, N.: Interlacing families II: Mixed characteristic polynomials and the Kadison-Singer Problem, arXiv:1306.3969

16. Mohar, B.L.: Median eigenvalues of bipartite subcubic graphs, Combin. Probab. Comput., in press. arXiv: 1309.7395 